\title{
ISOTOPIC CLOSED NONCONJUGATE BRAIDS ${ }^{1}$
}

\author{
K. MURASUGI AND R. S. D. THOMAS
}

ABstract. J. S. Birman has conjectured that, when a knot is represented by a closed braid on a minimal number $n$ of strands, the conjugacy class of the braid exhausts the set of braids in $B_{n}$ closing to define the knot. Counterexamples are given to disprove the conjecture, even when it is weakened to refer only to oriented knots.

1. Introduction. It is easy to see that a knot represented by the closure of a braid in the $n$-strand braid group $B_{n}$ can also be represented by the closure of a braid in $B_{m}$ for each $m>n$. Having made the above observation, J. S. Birman conjectured [1] that if $A$ is a knot and $n$ is the smallest integer such that there is a braid $\alpha \in B_{n}$ that closes to define the knot $A$, then the conjugacy class of $\alpha$ in $B_{n}$ gives the totality of braids in $B_{n}$ that close to define knots of the same isotopy type as $\alpha$. Leading to this conjecture is a proof that turning over a braid leads to a conjugate braid. Let $B_{n}$ be presented thus:

$$
\left\langle\sigma_{1}, \sigma_{2}, \cdots, \sigma_{n-1} ; \sigma_{i} \sigma_{j}=\sigma_{j} \sigma_{i} \text { if }|i-j| \geqq 2, \sigma_{i} \sigma_{i+1} \sigma_{i}=\sigma_{i+1} \sigma_{i} \sigma_{i+1}\right\rangle ;
$$

then the turning-over operation $\triangle$ can be described thus

$$
\sigma_{i_{1}}^{\varepsilon_{1}} \sigma_{i_{2}}^{\varepsilon_{2}} \cdots \sigma_{i_{k}}^{\varepsilon_{k}} \stackrel{\Delta}{\rightarrow} \sigma_{n-i_{1}}^{\varepsilon_{1}} \sigma_{n-i_{2}}^{\varepsilon_{2}} \cdots \sigma_{n-i_{k}}^{\varepsilon_{k}} .
$$

Since the conjecture is trivially true for $n=2$, we consider only the case $n \geqq 3$. We show by Counterexample 1 that a second operation, turning upside down, may not lead to a conjugate braid. We define the second operation thus:

$$
\sigma_{i_{1}}^{\varepsilon_{1}} \sigma_{i_{2}}^{\varepsilon_{2}} \cdots \sigma_{i_{k}}^{\varepsilon_{k}} \stackrel{\nabla}{\longrightarrow} \sigma_{n-i_{k}}^{\varepsilon_{k}} \cdots \sigma_{n-i_{2}}^{\varepsilon_{2}} \sigma_{n-i_{1}}^{\varepsilon_{1}}
$$

It is obvious that if a braid $\alpha$ closes to define a knot $A$, then both the braids $\triangle \alpha$ and $\nabla \alpha$ close to define a knot of the isotopy type of $A$. The original conjecture must therefore be weakened to concern only oriented knots.

We show by Counterexample 2 that the weakened conjecture admits an infinite class of counterexamples within $B_{n}$ for each $n \geqq 4$.

Received by the editors June 15, 1971.

AMS 1970 subject classifications. Primary 55A25.

Key words and phrases. Braid group, closed braid, conjugate braid, knot, composite knot, oriented knot.

${ }^{1}$ Research was supported by the National Research Council grants A4034 and A7914. 
2. Counterexample 1. The knot $6_{3}$ in Reidemeister's table can be represented by the closure of braids in $B_{3}$ but not in $B_{2}$. We consider the braid $\gamma=\sigma_{1}^{-1} \sigma_{2}^{2} \sigma_{1}^{-2} \sigma_{2} \in B_{3}$, which closes to define $6_{3}$. And we show that $\nabla \gamma=\sigma_{1} \sigma_{2}^{-2} \sigma_{1}^{2} \sigma_{2}^{-1} \in B_{3}$ is not conjugate to $\gamma$. When $\gamma$ and $\nabla \gamma$ are expressed within the presentation of $B_{3}$ as $\left\langle a, b ; a^{3}=b^{2}\right.$, where $a=\sigma_{1} \sigma_{2}$ and $b=$ $\sigma_{1} \sigma_{2} \sigma_{1}$, they can be written thus:

$$
\gamma=\left(b a b a^{2} b a^{2} b a b a b a^{2}\right) a^{-18}, \quad \nabla \gamma=\left(a^{2} b a b a b a^{2} b a^{2} b a b\right) a^{-18} .
$$

Since the conjugacy classes in this normal form are just the cyclic permutations of the bracketed factors, the classes of $\gamma$ and $\nabla \gamma$ are apparenily distinct.

3. Counterexample 2. It will be observed that the construction of the counterexamples to the weakened conjecture depends on the oriented knots' being both composite and not representable by the closure of a braid of $B_{3}$. Within $B_{4}$, let $\alpha=\sigma_{1}^{m} \sigma_{2}^{n} \sigma_{3}^{p}$ and $\beta=\sigma_{1}^{m} \sigma_{2}^{p} \sigma_{3}^{n}$ with $m, n, p$ all different, odd, and at least three in absolute value. Let $A, B$ be the oriented knots defined by orienting from top to bottom and closing $\alpha$ and $\beta$ respectively. Both $A$ and $B$ can be formed by the composition of oriented torus knots of types $(2, m),(2, n),(2, p)$. By the commutativity of the composition of oriented knots, $A$ and $B$ are isotopic: we show that neither can be represented as the closure of an element of $B_{2}$ or $B_{3}$ and secondly that $\alpha$ and $\beta$ are not conjugate in $B_{4}$.

Suppose that $A$ is represented in $B_{n}$. Then the group of $A$ is generated by at most $n$ Wirtinger generators with $n-1$ defining relations. Therefore the length of the chain of ideals ${ }^{2}$ of $A$ is at most $n-1$. But the length of the chain of ideals of a composite knot is the sum of the lengths of the chains of ideals of its components by [2]. And the length of the chain of ideals of each of the three components of $A$ is one. Therefore $3 \leqq n-1$ which implies that $n \geqq 4$. The same argument holds for $B$.

To show that $\sigma_{1}^{m} \sigma_{2}^{n} \sigma_{3}^{p}$ is not conjugate to $\sigma_{1}^{m} \sigma_{2}^{p} \sigma_{3}^{n}$ in $B_{4}$, let $N$ be the normal closure of $\sigma_{1} \sigma_{3}^{-1}$ in $B_{4}$ and let $\phi: B_{4} \rightarrow B_{4} / N \cong B_{3}$ be the natural homomorphism. Then

$$
\phi\left(\sigma_{1}^{m} \sigma_{2}^{n} \sigma_{3}^{p}\right)=\sigma_{1}^{m} \sigma_{2}^{n} \sigma_{1}^{p} \quad \text { and } \quad \phi\left(\sigma_{1}^{m} \sigma_{2}^{p} \sigma_{3}^{n}\right)=\sigma_{1}^{m} \sigma_{2}^{p} \sigma_{1}^{n} .
$$

But $\sigma_{1}^{m+p} \sigma_{2}^{n}$ and $\sigma_{1}^{m+n} \sigma_{2}^{p}$ are not conjugate in $B_{3}$ since when they are closed they do not define isotopic links but rather the torus knots of type $(2, n)$ and $(2, p)$ respectively linked to an unknotted circle. Therefore $\alpha$ is not conjugate to $\beta$ in $B_{4}$.

The existence of a similar infinite family of counterexamples in $B_{n}$ for each larger $n$ is obvious. These examples further stress the need to treat

${ }^{2}$ For the definition, see [2, p. 259]. 
oriented rather than unoriented knots since $\alpha$ can be used in place of $\gamma$ in $\S 2$.

\section{REFERENCES}

1. J. S. Birman, Non-conjugate braids can define isotopic knots, Comm. Pure Appl. Math. 22 (1969), 239-242. MR 39 \#6298.

2. R. H. Fox, On the total curvature of some tame knots, Ann. of Math. (2) 52 (1950), 258-260. MR 12, 273.

Department of Mathematics, University of Toronto, Toronto, Ontario, CANADA

Department of Computer Science, University of Manitoba, WinNipeg, Manitoba, Canada

Current address (Murasugi): Department of Mathematics, Princeton University, Princeton, New Jersey 08540 